EGU2020-18114

https://doi.org/10.5194/egusphere-egu2020-18114

EGU General Assembly 2020

(c) Author(s) 2021. This work is distributed under

the Creative Commons Attribution 4.0 License.

\title{
First steps towards deriving rock magnetic and paleomagnetic data from subsets of magnetic grains in lavas using Micromagnetic Tomography
}

\author{
Lennart de Groot ${ }^{1}$, Karl Fabian ${ }^{2}$, Annemarieke Béguin ${ }^{1}$, Martha Kosters ${ }^{1}$, Roger Fu ${ }^{3}$, Richard \\ Harrison $^{4}$, Auke Barnhoorn ${ }^{5}$, and Tristan van Leeuwen ${ }^{6}$ \\ ${ }^{1}$ Utrecht University, Faculty of Geosciences, Paleomagnetic laboratory Fort Hoofddijk, Utrecht, Netherlands \\ (I.v.degroot@uu.nl) \\ ${ }^{2}$ NTNU Trondheim, Dept. of Geoscience and Petroleum, Trondheim, Norway \\ ${ }^{3}$ Harvard University, Dept. of Earth and Planetary Sciences, Cambridge, MA, USA \\ ${ }^{4}$ University of Cambridge, Dept. of Earth Sciences, Cambridge, UK \\ ${ }^{5} \mathrm{TU}$ Delft, Dept. of Geoscience and Engineering, Delft, Netherlands \\ ${ }^{6}$ Utrecht University, Faculty of Sciences, Mathematical Institute, Utrecht, Netherlands
}

Our understanding of the behavior of the geomagnetic field arises from magnetic signals stored in geological materials, e.g. lavas. Almost all experiments to determine the past state of the Earth's magnetic field use bulk samples (typically $1-10 \mathrm{cc}$ ) and measure their magnetic moment after series of laboratory treatments. Lavas, however, consist of mixtures of different iron-oxide grains that vary in size, shape, and chemistry. Some of these grains are good recorders of the Earth's magnetic field; others are not. Only a small amount of adverse behaved magnetic grains in a sample already hampers all classical experiments to obtain paleointensities; success rates as low as $10-20 \%$ are common, i.e. for $80-90 \%$ of all lavas vital information on paleointensities is lost before it can be uncovered.

Recently, we showed that it is possible to determine the magnetization of individual grains inside a synthetic sample using a new technique: Micromagnetic Tomography. The individual magnetizations of grains are determined by inverting scanning magnetometry data from the surface on the sample onto the known locations, sizes and shapes of the magnetic grains that are obtained from a microCT scan of the sample. The synthetic sample used for our proof-of-concept, however, was optimized for success: the dispersion of magnetic markers was low, and the magnetite grains had a well-defined grain size range. Furthermore, the scanning SQUID microscope used requires the sample to be at $4 \mathrm{~K}$, below the Verweij transition of the magnetite grains.

Here we present the first Micromagnetic Tomography results from natural samples. We used two magnetic scanning techniques that operate at room temperature, a Magnetic Tunneling Junction set-up and a Quantum Diamond Magnetometer, to acquire the magnetic surface scans from a Hawaiian lava and calculated magnetic moments of individual grains present. We show that it is 
possible to acquire rock magnetic information as function of grain size from these natural samples and reveal the first results of interpreting a paleomagnetic direction from selected subsets of grains in our samples. These are the first steps towards deriving rock magnetic and paleomagnetic information from subsets of known good recorders inside lava samples, a technique that will revolutionize our field of research. 